\title{
EFFECT OF AN EXTERNAL VORTEX ON THE UAV AERODYNAMIC PERFORMANCES
}

\author{
Warda BOUDAOUD, Tayeb YAHIAOUI, Bachir IMINE, Omar IMINE`
}

\begin{abstract}
In this present work, the CFD and wind tunnel are used to investigate the effect of an external vortex around an UAV in order to obtain initial estimates of lift and drag coefficients with flow velocity of 30 meters per second for various angles of attack. The model of SpalartAllmaras turbulence is used for the investigation of the complex flow around the UAV. The wind tunnel and CFD results are compared and appropriate error bands placed on the data.
\end{abstract}

Keywords: Numerical - experimental - aerodynamic - drag - lift - turbulence model.

\section{INTRODUCTION}

The Unmanned Aerial Vehicle is a small plane which flies in an autonomous way with only one virtual pilot on board. That does not mean that the plane is completely out of control once it leaves the track. The presence of a human being in the loop of control is essential. But the operator is on a station on the ground, either on ground, or at sea or in a control center. He can intervene at any time, examine the situation and the rules of engagement, and stop the mission if necessary. The numerical simulation in the field of aerodynamics is relatively recent research tools. The specialists raise the question of knowing if the field of aerodynamics is purely theoretical or numerical, they estimate however that it is theoretical, because of the many numerical tests necessary for the stage to insufficiencies of the knowledge of the calculation methods [1].

In this present work, a numerical and experimental study of the effect of external vortex around an UAV is presented. The generation of this external vortex is obtained using an isolated wing and placed before the UAV of a distance $C$ as it shown in the figure 1 and subjected to an incidence of 10 degrees. When the flow crosses this isolated wing a tip vortex is generated on the level of the end of wing and disturbs the flow around the UAV. The first phase of this work consists in using plans of construction of this UAV to create a solid model which specifies its external geometry in Solid Works (Figure1).

- Laboratory of aeronautics and propulsive systems - Mechanical Engineering Faculty, USTO Oran, B.P 1505 El Mnaouer U.S.T. Oran. Algeria;E-mail: imine_b@yahoo.fr 
This solid model is then exported in to a programme of the grid generation in order to return its geometry adapted to aerodynamic calculations and tested in various flying conditions [2]. A computer code of CFD [3] is used to obtain estimates of the coefficients of lift and drag to 30 meters per second. The model of turbulence Spalart-Allmaras [4] is used to leap

the transport equations of Navier-Stokes in order to correctly predict the complex flow around the plane. A study of the independence of the grid is then carried out to determine the precision of the grid of calculation used for analysis CFD. The second phase of this work consists for creating a 1/6th scale wind tunnel model. Wind tunnel testing is conducted at 30 meters per second and many various angles of attack are used in order to obtain lift, drag, and moment coefficient data. The wind tunnel and CFD results are compared and appropriate error bands placed on the data.

\section{DESCRIPTION OF THE UAV}

This UAV as shown in Figure 2 is 2 meters long and provided with a pendular wing of the scale of $3 \mathrm{~m}$. the profile of this wing is of type Clark yh with a cord of $0.236 \mathrm{~m}$, stalled on the fuselage of $4^{\circ}$ with a dihedral of $4^{\circ}$. The empennage group of the shape of $\mathrm{V}$ reversed is profiled with Naca 0012 and attached to the wings by two beams which give also the name of an UAV to twin-boom. These UAV's aspire of a remarkable stability to the no desirable movements of roll. This UAV is designed to have a pusher engine which will be embarked behind the fuselage.

\section{TRANSPORT EQUATIONS AND TURBULENCEMODEL}

The flow around the plane is considered turbulent asymmetrical. The general forms of the transport equations can be written in Cartesian coordinates as:

Continuity equation

$$
\frac{\partial}{\partial \mathrm{x}_{\mathrm{j}}}\left(\bar{\rho} \widetilde{\mathbf{U}}_{\mathrm{j}}\right)=0
$$

Momentum conservation equation

$$
\frac{\partial}{\partial x_{j}}\left(\bar{\rho} \widetilde{U}_{i} \widetilde{U}_{j}\right)=\bar{\rho} g_{i}-\frac{\partial \bar{p}}{\partial x_{i}}-\frac{\partial}{\partial x_{j}}\left(\rho_{u} i^{\prime \prime} u_{j}{ }^{\prime \prime}\right)+\frac{\partial}{\partial x_{j}}\left[\bar{\mu}\left(\frac{\partial \widetilde{U}_{i}}{\partial x_{j}}+\frac{\partial \widetilde{U}_{j}}{\partial x_{i}}\right)-\frac{2}{3} \bar{\mu} \frac{\partial \widetilde{U}_{k}}{\partial x_{k}} \delta_{i j}\right]
$$

\section{Turbulence model}

The model of turbulence used in this present work is a model with a transport equation for the $v$ quantity suggested by Spalart and Allmaras [3]:

$$
\frac{\partial(\bar{\rho} \widetilde{v})}{\partial t}+\vec{\nabla} \cdot(\bar{\rho} \widetilde{\tilde{V}} \widetilde{v})=\vec{\nabla} \cdot\left[\frac{\bar{\mu}+\bar{\rho} \widetilde{v}}{\partial S A} \vec{\nabla} \widetilde{v}\right]+P_{v}-D v
$$

Or the terms of production and destruction are defined as follows: 


$$
\begin{gathered}
\mathrm{P}_{v}=C_{b 1} C_{\mu} \widetilde{\mathrm{S}} \tilde{\rho} \widetilde{\mathrm{v}}+C_{b 2} \frac{\bar{\rho}(\vec{\nabla} \widetilde{v})^{2}}{\partial S A} \\
D_{v}=C^{\prime} \omega 1 f_{\omega} \frac{\bar{\rho}(\vec{\nabla} \widetilde{v})^{2}}{\rho d^{2}}
\end{gathered}
$$

With

$$
f_{x}=g\left(\frac{1+C_{\omega 3}^{6}}{g^{6}+C_{\omega 3}^{6}}\right)^{1 / 6} \text { With } g=r+C \omega 2\left(r^{2}-r\right)
$$

And

$$
M_{t}=\bar{\rho} \widetilde{v} \frac{\chi^{3}}{\chi^{3}+C_{v 1}} \chi=\frac{\bar{\rho} \widetilde{v}}{\mu}
$$

(For more information see reference 3)

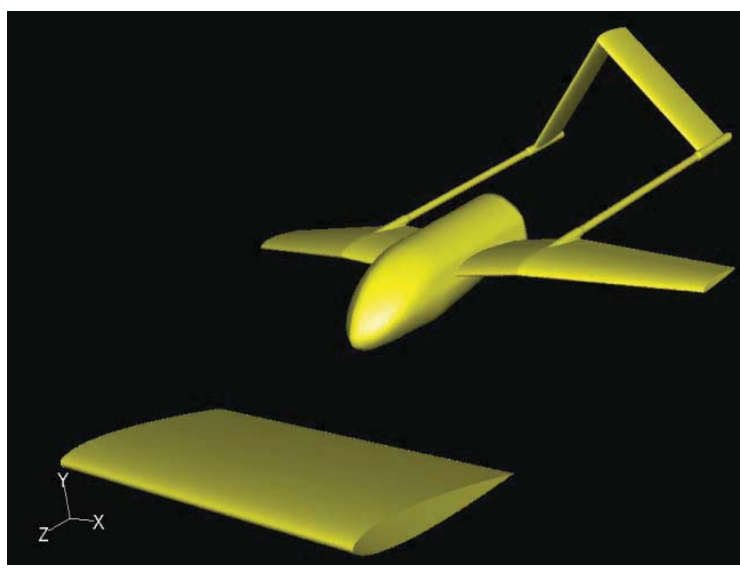

Figure 1: Computational model

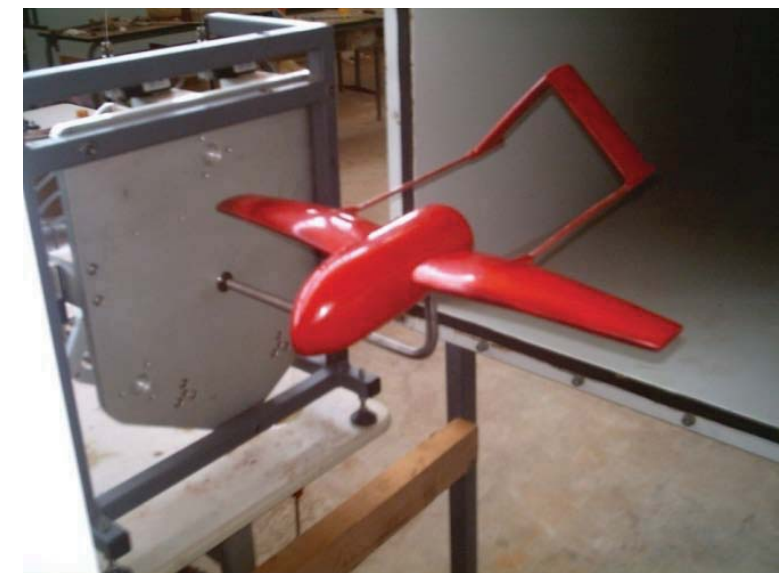

Figure 2: Experimental model

\section{EXPERIMENTAL FACILITY}

The Wind Tunnel used is of a closed circuit, horizontal return type. The Closed Circuit Wind Tunnel is of conventional design and has advantages over a similar open circuit design (see Figure 3). These include; a higher maximum velocity, lower power consumption and lower noise level. It is driven by an A.C motor and axial flow fan that forces air around the circuit and produces a maximum velocity of $60 \mathrm{~m} / \mathrm{s}$. In order to conduct wind tunnel testing of the UAV, a prototype was designed from plans drawn in SolidWorks. The prototype is made in wood to a scale of $1 / 6$ for a span of $50 \mathrm{~cm}$ corresponding to the maximum width of the test section of wind tunnel. 


\section{MESHING AND BOUNDARY CONDITIONS}

As most problems involved with fluid flow, a setup that would constitute a virtual wind tunnel was ideal. All of the three-dimensional problems could be divided along the longitudinal axis for symmetry. After the models were imported from the SolidWorks, a box was created which surrounded the whole of the model and gave sufficient room around the object to analyze the flow, yet was tight enough to lower the number of internal nodes to a minimum.

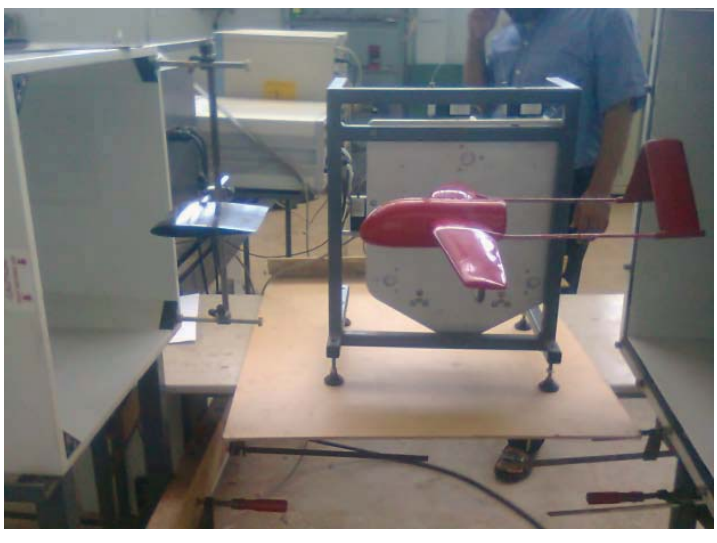

Figure 3: Wind tunnel

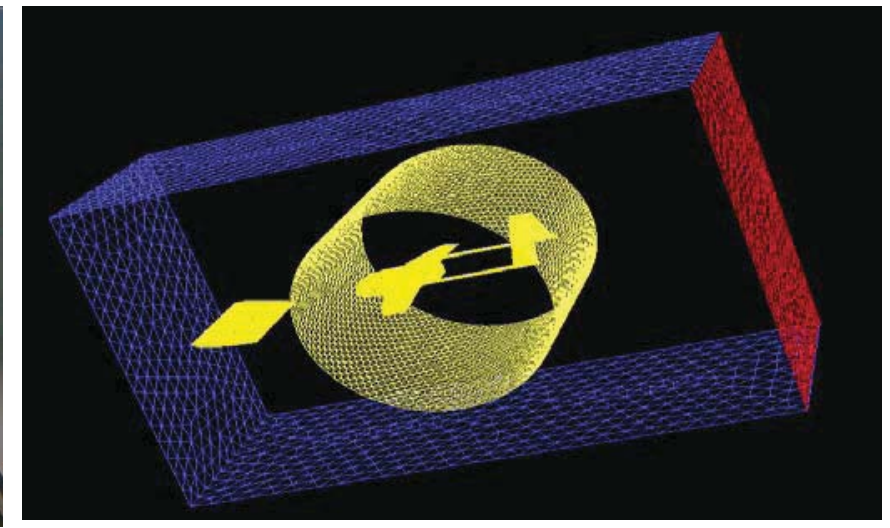

Figure 4: Meshing

As it shown in figure 4, typically, the fluid box was modelled to have one inlet and it composed on two blocks: a rotating one containing the UAV to carry out the various incidences inside a fix block who contained the isolated wing. The geometry itself consisted of non-slip interior boundaries. The box also provided easy boundary specifications for the structural problems. Additionally, this method allowed easy estimation of the drag forces produced by the various components, if desired.

\section{RESULTS}

Several compilations and measures were carried out for different angles of attack to determine the lift and drag. Each case was carried out at the same speed: 20 meters per second. The angle of attack extended from -10 to 17 degrees.

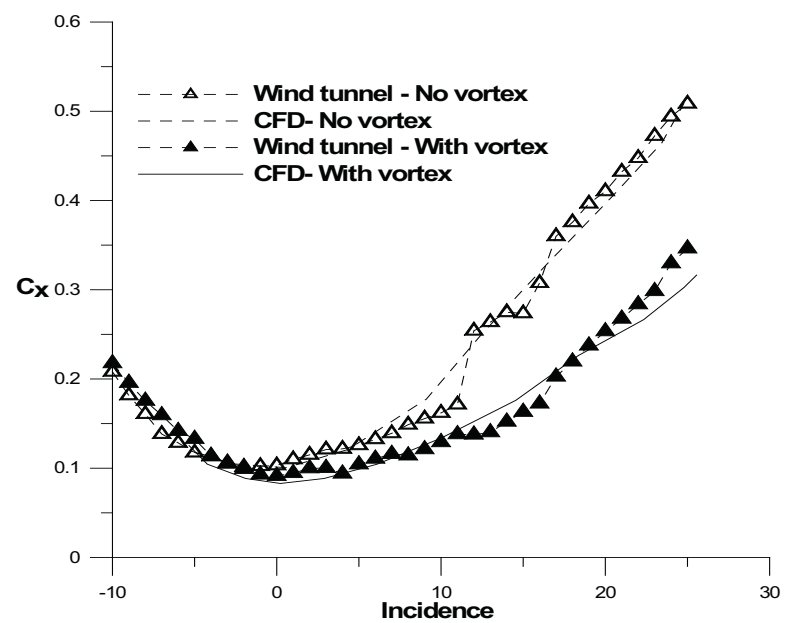

Figure 5: Evolution of the drag coefficient

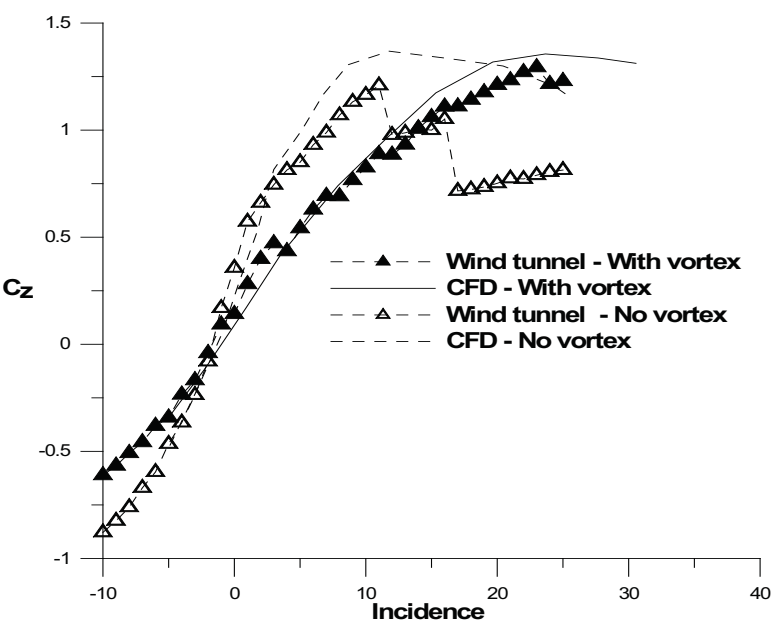

Figure 6: Evolution of the lift coefficient 
The evolution of the drag coefficient according to the angle of attack is presented in figure 5 in the experimental and numerical cases. It is a parabolic type tendency similar to those found on the planes in general. In particular, one notes the increase in the drag with the increase in the angle of attack. In general, the numerical and experimental curves follow the same tendency separately which is normal because variation of the drag between the two curves corresponds to the drag of the remainder of the UAV which varies very slightly with the incidence.It's clear the influence off the vortex is visible UAV and especially for positive incidences where one notes a remarkable reduction of the drag. On the other hand for negative incidences one notes that the vortex develops with the top of the UAV without influencing its drag

Figure 6 compares the computational evolution of the lift according to the angle of attack for the UAV with the experimentally lift. An agreement of the two curves is noted in the linear zone. However, examining the stall zone, the differences between the numerical study and deviations from the measured curve become visible. It is clear that the numerical simulation is far from predicting the stall, because the models currently used are not accurate for flows with separate streams. Based on this comparison, it was concluded that the limited use of CFD is the post-stall is to say, at angles of attack below $14^{\circ}$ or less [4]. Experimental measurement show well the phenomenon of the UAV stall which is located at approximately $12^{\circ}$. It is noted that the presence of the vortex largely delays the stall of the UAV from $12^{\circ}$ at $24^{\circ}$.

The computational pressure distribution around the UAV is represented in figures 7 . One notices in the cases $\mathrm{i}=0^{\circ}$ that there exists a dissymmetry in the distribution of the computational total pressure around the UAV. This dissymmetry is justified by the effect of the same vortex for a null incidence. Therefore, this dissymmetry generates an instability on the $\mathrm{f}$ roll axis. Figure 8 shows the creation of the vortex of end of wing which appears with the front of the UAV.

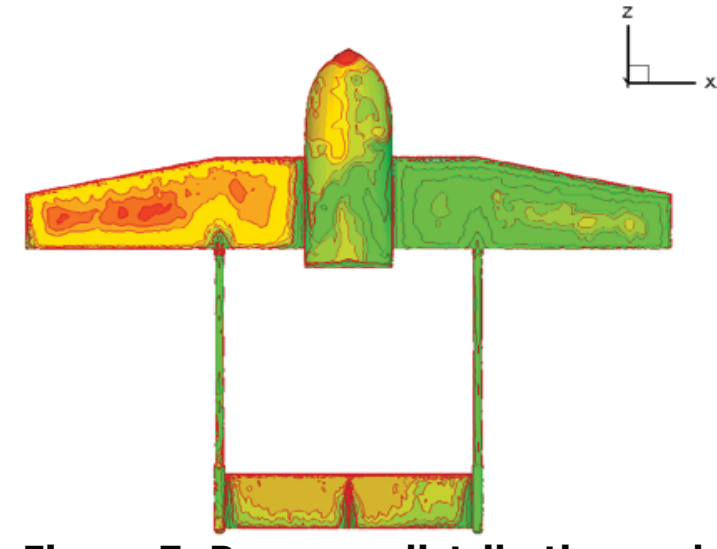

Figure 7: Pressure distribution and streamlines for $i=0^{\circ}$

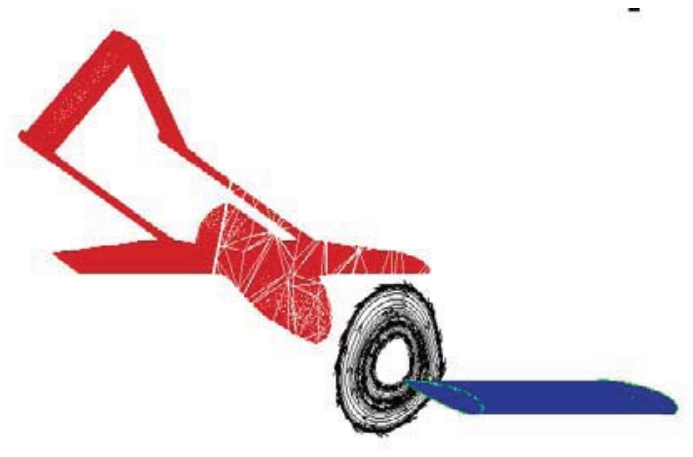

Figure 8: Computational capture of tip vortex

In Figures 9 and 10, the vortical structures in the aft region of the UAV and in the wake are visualized which release from salmons of the wing to the passage of the flow for the incidence $10^{\circ}$ and $15^{\circ}$. The streaky structures in the near wall region are elongated near the trailing edge and spanwise vortices form and grow in the wake region. Moreover, one 
notes the complexity of the flow around the plane for an incidence judged like the beginning of stall.

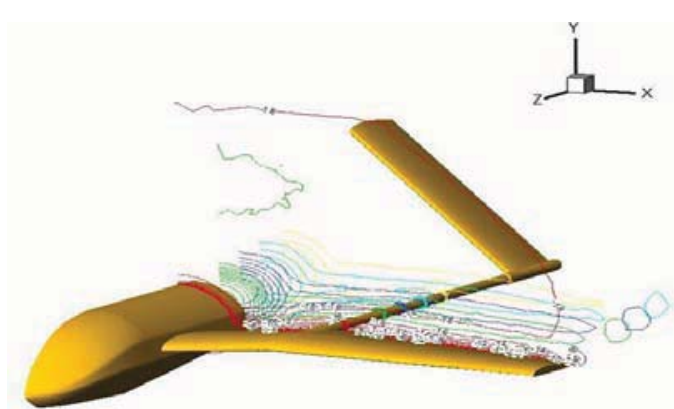

Figure 9: Vortical structures

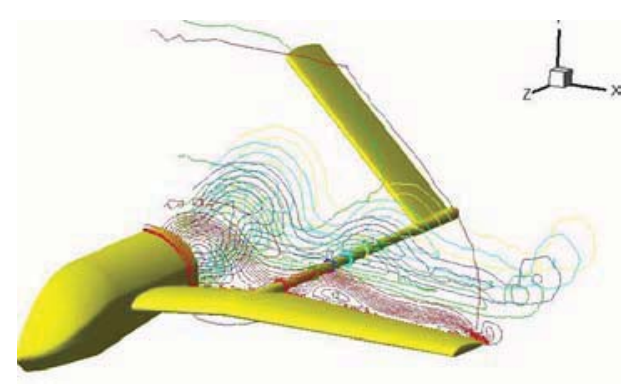

Figure 10: Vortical structures

\section{CONCLUSIONS}

The present study was undertaken to observe the effect of an external vortex on the aerodynamic performances of an UAV by using a CFD computer code and an experimental investigation. The overall agreement of the numerical results with the experimental data is reasonable. The results show that the influence of the vortex is largely noted around the UAV and the phenomena of instability around the roll axis is visible.

\section{REFERENCES}

[1] Ronzheimer A.: Post-parameterization of CAD-geometries using freeform deformation and grid generation techniques. Notes on Numerical Fluid Mechanics and Multidisciplinary Design, Vol. 87, 2005, p. 382-389.

[2] Flores J., Reznick S.G., Hol T. and GundyK.: Transonic Navier-Stokes Solutions for a Fighter-Like Configuration. Journal of Aircraft 25, October 1988, p. 875-881.

[3] Bhaskaran B.: FLUENT Short Course, http://instruct1.cit.cornell.edu/courses/fluent. Includes step-by-step tutorials and problem sets. Chapters: Introduction to Computational Fluid Dynamics; Laminar pipe flow; Turbulent pipe flow; Compressible nozzle flow; Airfoil flow.

[4] Wallin S., Johansson A.V.: An explicit algebraic Reynolds stress model for incompressible and compressible turbulent flows", Journal of Fluid Mechanics 403 (2000) p. 89-132. 